\title{
Guided Wave Experimentation using CLoVER Transducers for Structural Health Monitoring
}

\author{
Ken I. Salas* and Carlos E. S. Cesnik ${ }^{\dagger}$ \\ Active Aeroelasticity and Structures Research Laboratory \\ Department of Aerospace Engineering, The University of Michigan, Ann Arbor, MI, 48109, USA
}

\begin{abstract}
The guided wave field excited by a wedge-shaped anisotropic piezocomposite transducer is experimentally investigated with applications to large area structural health monitoring (SHM). This investigation supports the experimental characterization of the Composite Long-range Variable-direction Emitting Radar (CLoVER) transducer introduced by the authors. The multiple advantages of this device over alternative damage interrogation concepts are outlined, and its manufacturing procedure is briefly reviewed. The experimental studies include both piezoelectric-based frequency response as well as laser vibrometry measurements in pristine isotropic structures. The results are compared with predictions from an analytical model. A comprehensive set of experimental results is presented that validate the accuracy of the solution in the time, frequency, and spatial domains. The damage interrogation approach proposed with this transducer concept is also experimentally demonstrated using simulated structural damage.
\end{abstract}

\section{Nomenclature}

A Area

$A^{*} \quad$ Aspect Ratio

$b \quad$ Substrate half thickness

$c_{g} \quad$ Group velocity

d Radial distance

$D$ Dispersion equation for Rayleigh-Lamb waves

$E, \nu$ Young's modulus and Poisson's ratio

$g_{i j} \quad$ Piezoelectric constants $(i, j=1,2,3)$

$r, \theta \quad$ Radial/Azimuthal coordinates

$\Delta r \quad$ Radial dimension

$R_{O} \quad$ Transducer's outer radius

$R_{I} \quad$ Transducer's inner radius

$S(\omega)$ Fourier transform of toneburst signal

$T \quad$ Excitation pulse period

$t \quad$ Time

$\bar{t} \quad$ Thickness

u Displacement vector

$w^{*} \quad$ Non-dimensional sensor width

$x, y \quad$ Cartesian coordinates

$\boldsymbol{\Gamma}, \boldsymbol{\Psi}$ Coefficient matrices

$\Delta \theta \quad$ Azimuthal dimension

$\epsilon_{i j} \quad$ Strain components $(i, j=x, y$ or $r, \theta)$

$\xi \quad$ Radial wavenumber

$\rho \quad$ Substrate material density

\footnotetext{
${ }^{*}$ Graduate Research Assistant, Student Member, AIAA; Corresponding Author; Email: ksalas@umich.edu; Ph: 734-9360541 .

${ }^{\dagger}$ Associate Professor and Associate Fellow, AIAA; Email: cesnik@umich.edu; Ph: 1-734-764-3397; Fax: 1-734-763-0578.
} 
$\phi \quad$ Azimuthal wavenumber

$\omega \quad$ Angular frequency

$\omega_{0} \quad$ Center frequency of toneburst signal

Subscript

a Actuator

A Antisymmetric mode

$h \quad$ Hole

$r \quad$ Reflection

$s \quad$ Sensor

$p \quad$ Excitation pulse

\section{Introduction}

Structural health monitoring (SHM) is the component of damage prognosis systems responsible for interrogating a structure's condition to identify, locate, and characterize any damage present. Among the different SHM schemes available, guided wave (GW) testing methods are very attractive due to the active nature of GWs, their ability to propagate through the thickness as well as over the surface, and their sensitivity to different damage types. In this approach, a GW is excited using a surface bonded transducer, and recorded by sensors distributed over the structure. Signal processing algorithms are later used along with the recorded signal to determine whether damage is present, and if so, its location and type. A comprehensive review of this SHM approach was presented by Raghavan and Cesnik. ${ }^{1}$

The use of an effective damage interrogation approach and the efficient excitation of GWs are two important aspects of a SHM system architecture. The damage interrogation approach must be able to efficiently cover the entire structural area from a small number of central locations. Consequently, the number of transducers employed should be sufficient to achieve this, but small enough to avoid a system with high power requirements. Moreover, a small number of transducers prevents intricate installation and maintenance procedures. In the context of a SHM system, the efficient excitation of GWs is related to the transducer's ability to generate directional wave fields that minimize losses by concentrating the actuation along a desired direction. In the realm of piezoelectric-based actuation, anisotropic piezocomposite transducers $(\mathrm{APT})^{2,3}$ are well suited for this application due to their ability to produce focused strain fields. These devices function under the 3-3 piezoelectric effect, where the polarization and desired actuation directions are parallel. This feature is achieved through the use of interdigitated electrodes acting over a piezoceramic fiber sheet. Additional benefits of these transducers over conventional piezoceramic wafers include larger strain energy density and enhanced surface conformability. In spite of their multiple advantages, conventional APT configurations are not able to simultaneously achieve these objectives as these either cover a reduced structural area (rectangular geometry), or must scan every direction simultaneously (circular or ring geometries).

An alternative approach is the use of phased arrays, where several actuators (either APT or piezoelectric wafers) are excited with known time delays to form a scanning beam by using signal interference (enforcing the signal along one direction and destroying it along the remaining ones). Several researchers have explored this approach in the past. For example, Wilcox et. al. ${ }^{4}$ used circular and linear arrays for the inspection of large areas of thick plate-like structures. Their study used both piezoelectric discs and shear piezoelectric transducers, and demonstrated that the ratio of the area covered to the array dimension was as large as 3000 to 1 . Further efforts along this direction were presented by Fromme et. al. ${ }^{5}$ who developed a ring array of piezoelectric transducers bonded on plate-like structures using a protective membrane. This array was attached to the structure in a compact housing that included the necessary multiplexing electronics, and was successfully used in detecting simulated corrosion and through-hole damage. This concept has also been successfully realized experimentally by Giurgiutiu et. al., ${ }^{6,7}$ yielding very narrow GW scanning beams that were successful in identifying cracks. However, it was reported that the increased number of transducers needed produced wiring difficulties. This approach has also been extended to the case of composite laminated structures by Zemmour and Pines. ${ }^{8}$ Although this type of array is able to scan every direction independently, it requires a large number of actuators and does not use the excitation energy efficiently.

We have presented a new transducer concept for efficient GW excitation and damage interrogation from a central location. ${ }^{9,10}$ The Composite Long-range Variable-direction Emitting Radar (CLoVER) transducer, shown in Fig. 1(a), has an overall ring geometry, but is composed of wedge-shaped APT sectors that can be 
individually excited to interrogate the structure along a particular direction. By sequentially exciting each CLoVER sector, the complete structural area can be covered. A brief review of this transducer's advantages and its manufacturing procedure is presented in a subsequent section.

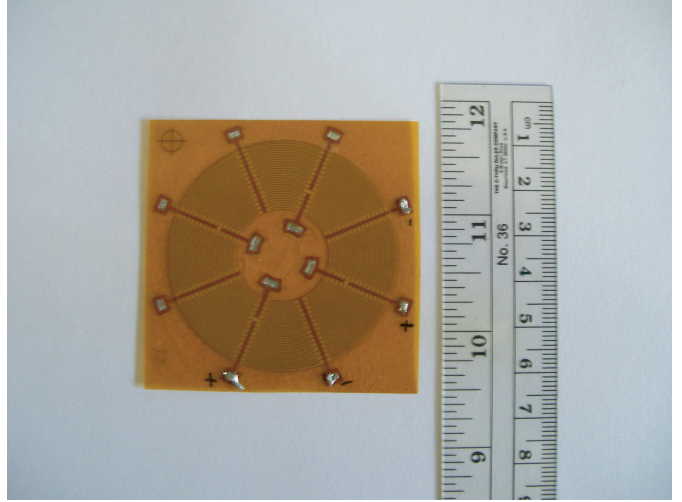

(a)

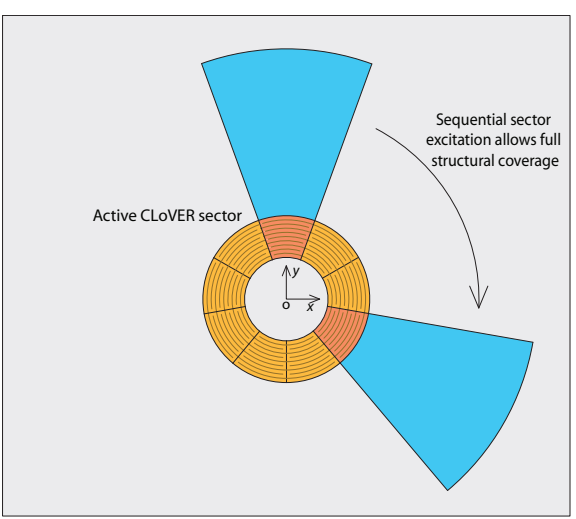

(b)

Figure 1. (a) Illustration of the CLoVER transducer; (b) Schematic showing interrogation approach proposed with CLoVER transducers.

The objectives of the present study are to experimentally characterize the performance of an individual CLoVER sector, and to use these results in verifying the theoretical formulation presented previously by the authors $^{11}$ for GW excitation by this transducer geometry. In addition, the damage interrogation approach proposed with CLoVER transducers is demonstrated experimentally. GW experiments directed towards SHM applications have been performed in the past by several researchers. In general, two approaches have been used to record the GW field generated experimentally. The first consists of bonding piezoelectric sensors on the surface of the structure under investigation to record the strain field produced by the actuator. Using this approach, the sensor can be sized so that it is insensitive to specific Lamb wave modes which is a desirable feature for damage detection. An additional benefit is that there is no limit to the Lamb wave propagation frequency that can be sensed. Some important disadvantages of this approach are the fact that the sensor's performance is susceptible to environmental conditions, such as electromagnetic interference (EMI), and that information is only recorded at the point where the sensor is placed. Raghavan and Cesnik have used this approach successfully in sensing the GW field generated by piezoelectric-based actuators in pristine and damaged isotropic structures. ${ }^{12-14}$ More recently, this was used to study the effects of environmental conditions, such as elevated temperatures, on GW propagation and damage detection. ${ }^{15}$

The second approach is based on the non-contact technique of laser vibrometry, where a laser beam is used to record the out-of-plane velocities induced by the piezoelectric actuator using the Doppler shifting phenomenon. This approach allows outstanding visualization of the wave field, which is valuable in studying its interaction with different damage types. However, this method is impractical for on-line based inspection, and post-processing of the data recorded (smoothing and denoising) is usually necessary before it can be effectively analyzed. ${ }^{16}$ Staszewski et. al. have used this method to study the GW field excited by piezoelectric transducers in metallic structures. Their initial studies successfully verified this technique's performance in sensing low-frequency $(75 \mathrm{kHz}) \mathrm{GWs}$ in isotropic plates. ${ }^{16}$ Their investigation was later directed towards detecting different damage types such as circular holes, rectangular notches, and cracks. ${ }^{17,18}$ More recently, they have used 3D laser vibrometry to directly measure all displacement components with applications to fatigue crack detection. ${ }^{19}$

The present study uses a combination of these two approaches to obtain a thorough description of the GW field excited by an individual CLoVER sector. The paper begins with a description of the CLoVER transducer where its salient features and manufacturing procedure are discussed. This is followed by a review of the theoretical model for GW excitation by a CLoVER sector. The experimental procedures are subsequently described, followed by comparisons between the experimental results and the model predictions. The paper concludes with a preliminary demonstration of the damage interrogation approach proposed with CLoVER transducers. 


\section{The CLoVER Transducer}

\section{A. Description and Advantages}

The CLoVER transducer has been introduced ${ }^{9,10}$ as an alternative concept for efficient damage interrogation and GW excitation in SHM systems. This transducer consists of a collection of wedge-shaped APTs arranged in a circular array that are individually activated to scan a complete $360^{\circ}$ structural range.

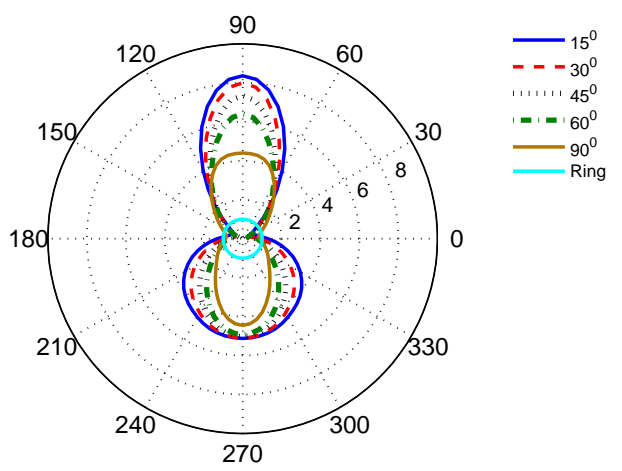

(a)

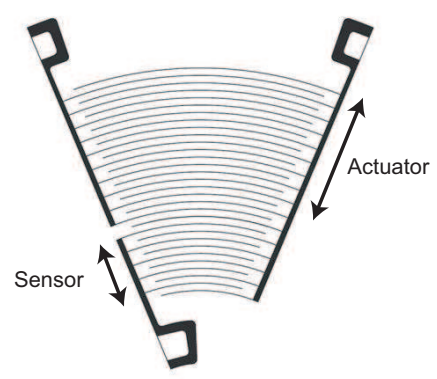

(b)

Figure 2. (a) Azimuthal distribution of out-of-plane displacement for different CLoVER azimuthal spans; (b) Electrode design used in first generation of CLoVER sectors.

Previous work by the authors ${ }^{11}$ has shown that the CLoVER transducer presents several improvements over alternative concepts. First, the amplitude of the displacements induced by a CLoVER sector can be increased by at least $50 \%$ from those obtained using a ring configuration for similar electric current inputs. This is shown in Fig. 2(a) where the out-of-plane displacement for CLoVER sectors with different azimuthal spans are compared at a fixed radial position, and a frequency-thickness product of $208 \mathrm{kHz}-\mathrm{mm}$. The displacements for each sector have been normalized by those of a similarly-sized ring configuration. The figure shows that if each CLoVER sector is provided with the same electric current input as the ringshaped transducer, the displacements obtained can be increased by as much as a factor of 8 , for the specific parameters under consideration.

A desirable feature for detecting damage using GWs is that the transducer be capable of selectively exciting individual Lamb modes. It is well known that different modes are necessary to identify a wide spectrum of damage types. ${ }^{20,21}$ For instance, the fundamental symmetric $\left(\mathrm{S}_{0}\right)$ mode is preferred for throughthe-thickness defects, while the fundamental antisymmetric $\left(\mathrm{A}_{0}\right)$ mode is more sensitive to surface damage. The interdigitated electrode design used in the first generation of CLoVER transducers uses two independent radial subdivisions that can be used to achieve modal selectivity. This can be achieved by selecting the radial dimension according to the wavelength of the desired mode. In addition, these give the transducer the ability to independently act as an actuator and sensor, as schematically shown in Fig. 2(b), thereby decreasing the number of transducers needed for inspection. Finally, the composite construction characteristic of APTs gives the CLoVER transducer the ability to conform to curved surfaces (e.g., aircraft fuselages), an increased resistance to environmental damage, and a higher specific strength than monolithic piezoelectric wafers.

\section{B. Actuator Manufacture}

The CLoVER transducers used in this study were manufactured based on an adaptation of the procedure presented by Wilkie et. al. ${ }^{22,23}$ for building Macro-Fiber Composite (MFC) actuators. The design and construction process is outlined in Fig. 3. The first step consisted of designing the desired interdigitated electrode pattern using a suitable CAD application. Once the design was completed, the electrode pattern was printed on a copper-clad kapton film (Pyralux LF7062R) using photolithography (MetroCircuits Inc). The devices used in this study had an electrode finger width of $0.1 \mathrm{~mm}$ and an electrode finger spacing 
of $0.5 \mathrm{~mm}$, both parameters consistent with those used in NASA-standard MFCs. PZT- 5A piezoceramic rings with $0.2 \mathrm{~mm}$ thickness (EBL Products) were diced into wedge-shaped fibers with a cut angle of two degrees (American Dicing), so that a fiber width of $0.36 \mathrm{~mm}$ was achieved at the inner radius. This value corresponds to the width of prismatic piezoceramic fibers used in typical APT devices. ${ }^{24}$ The fibers and electrodes were bonded using an epoxy adhesive system (Hysol Loctite E-120HP).

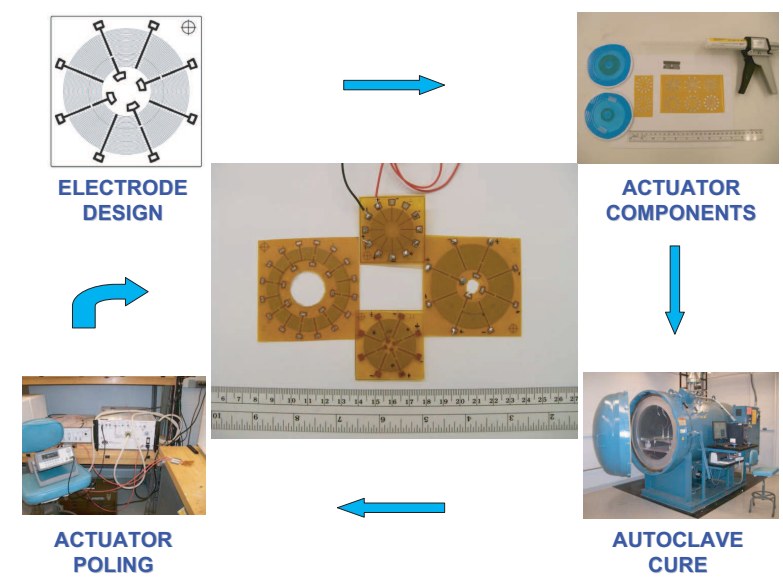

Figure 3. CLoVER transducer development stages.

The transducers were cured in an autoclave, while the standard manufacturing procedure employed a vacuum hot press for this purpose. Consequently, the cure cycles presented by Wilkie et. al. ${ }^{22}$ were used as a starting point, and fine tuned for the autoclave cure. Each actuator was cured for two hours in a vacuum $(-28.2 \mathrm{in} \mathrm{Hg})$ bag at $100 \mathrm{psi}(689.5 \mathrm{kPa})$ and $250{ }^{\circ} \mathrm{F}\left(121{ }^{\circ} \mathrm{C}\right)$. This cure time and temperature have been previously shown to be sufficient for full actuator cure based on experimentally-calibrated cure kinetics models. ${ }^{23}$ A new poling procedure was also employed which resulted in transducers with performance levels similar to those of NASA-standard MFCs. Further details of the manufacturing and characterization procedures can be found in an earlier work by the authors. ${ }^{10}$

\section{Review of GW excitation by a CLoVER sector}

An analytical model for the GW field excited by a CLoVER sector was presented in a previous work by the authors. ${ }^{11}$ The description of the problem considered an infinite isotropic substrate, and used the assumptions of perfect bonding and uncoupled substrate and actuator dynamics. As a result, their interaction was modeled through shear tractions along the transducer's radial edges on the substrate's surface. The problem was formulated using the 3-D equations of elasticity and the solution was found using Fourier transforms and complex variable techniques. The displacement field induced by the wedge-shaped APT was presented for three separate spatial regions according to the expected wave behavior, as shown in Fig 4(a). Region III was characterized by the presence of outward propagating waves originating from both actuator edges, while region I only considered inward propagating waves. Region II considered waves propagating inwards originating from the actuator's outer edge, and outward propagating waves from the inner edge.

For the present experimental verification studies, only the displacement field induced in region III will be necessary. For this region, the time-dependent Cartesian displacement components for the antisymmetric mode, expressed in polar coordinates $r$ and $\theta$, are given by: ${ }^{11}$

$$
\mathbf{u}_{A}(r, \theta, t)=\frac{1}{2 \pi} \int_{-\infty}^{\infty} \sum_{\xi_{A}}\left[\sum_{k=-\infty}^{\infty} \frac{i \pi^{2} \boldsymbol{\Gamma}_{A}\left(\xi_{A}\right)}{\zeta_{k}(\theta) D_{A}^{\prime}\left(\xi_{A}\right)} \boldsymbol{\Delta}_{A}\left(k, \xi_{A}\right) H_{k}^{(2)}\left(\xi_{A} r\right)\right] S\left(\omega, \omega_{0}\right) e^{i \omega t} d \omega
$$

where $\xi_{A}$ represents the propagating wavenumber for the antisymmetric mode, $\boldsymbol{\Gamma}_{\mathbf{A}}$ and $\boldsymbol{\Delta}_{\mathbf{A}}$ are coefficient vectors associated with region III, and $D_{A}^{\prime}$ represents the derivative of the Rayleigh-Lamb dispersion equation for antisymmetric modes with respect to the wavenumber, $\xi$. The outward propagating radiation pattern is introduced by the Hankel function of the second kind, $H_{k}^{(2)}\left(\xi_{A} r\right)$. The term $S\left(\omega, \omega_{0}\right)$ corresponds to the 


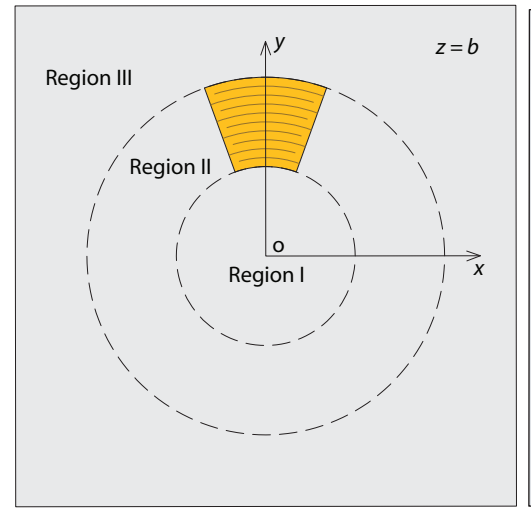

(a)

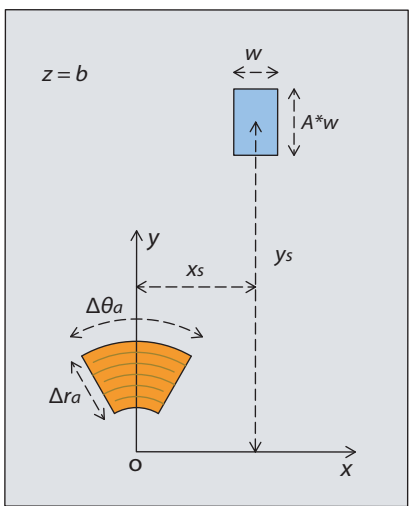

(b)

Figure 4. (a) The domain is divided into three spatial regions according to wave behavior; (b) Geometry used in rectangular sensor response analysis.

Fourier transform of the time-dependent part of the excitation which, for SHM applications, is typically given by a Hann-modulated toneburst at a center frequency $\omega_{0}$.

The displacement field given by Eq. (1) can be used to obtain the response of a piezoelectric sensor under the GW field excited by the CLoVER sector. Consider a rectangular sensor centered at the point $\left(x_{s}\right.$, $y_{s}$ ) with width $w$ and aspect ratio $A^{*}$, so that its surface area, $A_{s}$, is given by $A^{*} w^{2}$ as shown in Fig. 4(b). Using plane stress conditions and modeling the sensor as a parallel plate capacitor, ${ }^{13}$ the voltage it generates due to the induced strains is given by:

$$
V_{s}=\frac{1}{2 \pi} \int_{-\infty}^{\infty} \frac{E_{s} \bar{t}_{s} g_{13}}{A_{s}\left(1-\nu_{s}\right)} \int_{y_{s}-A^{*} \frac{w}{2}}^{y_{s}+A^{*} \frac{w}{2}} \int_{x_{s}-\frac{w}{2}}^{x_{s}+\frac{w}{2}}\left(\epsilon_{x x}+\epsilon_{y y}\right) d x d y S\left(\omega, \omega_{0}\right) e^{i \omega t} d \omega
$$

where $E_{s}$ represents the sensor's Young's modulus, $\nu_{s}$ represents the sensor's Poisson's ratio, $\bar{t}_{s}$ represents the sensor thickness, and $g_{13}$ represents the piezoelectric constant. The in-plane normal strains produced by the actuator are denoted by $\epsilon_{x x}$ and $\epsilon_{y y}$. These components are given by:

$$
\begin{aligned}
\epsilon_{x x} & =\frac{\partial u_{1}}{\partial r} \cos \theta-\frac{\partial u_{1}}{\partial \theta} \frac{\sin \theta}{r} \\
\epsilon_{y y} & =\frac{\partial u_{2}}{\partial r} \sin \theta+\frac{\partial u_{2}}{\partial \theta} \frac{\cos \theta}{r}
\end{aligned}
$$

where the derivatives of the displacement components can be found from Eq. (1) as:

$$
\frac{d \mathbf{u}_{A}}{d r}=\sum_{k=-\infty}^{\infty}\left[\frac{i \pi^{2} \xi_{A} \boldsymbol{\Gamma}_{A}\left(\xi_{A}\right)}{\zeta_{k}(\theta) D_{A}^{\prime}\left(\xi_{A}\right)} \boldsymbol{\Delta}_{A}\left(k, \xi_{A}\right)\left\{H_{k-1}^{(2)}\left(\xi_{A} r\right)-H_{k+1}^{(2)}\left(\xi_{A} r\right)\right\}\right]
$$

and:

$$
\frac{d \mathbf{u}_{A}}{d \theta}=\sum_{k=-\infty}^{\infty}\left[\frac{-2 k \pi^{2} \boldsymbol{\Gamma}_{A}\left(\xi_{A}\right)}{\zeta_{k}(\theta) D_{A}^{\prime}\left(\xi_{A}\right)} \boldsymbol{\Delta}_{A}\left(k, \xi_{A}\right) H_{k}^{(2)}\left(\xi_{A} r\right)\right]
$$

The result of Eq. (2) is a time domain signal for each center frequency, $\omega_{0}$. The frequency response results to be compared with the experimental data are obtained by finding the peak-to-peak value of this signal over a given range of center frequencies. 


\section{Sensor-based Experiments}

In this section, piezoelectric sensors are employed to verify the accuracy of the theoretical solution in the frequency domain. This method is restricted to single-point measurements, but it is able to provide information on the performance of the transducer over a wide frequency range. This is a critical component of the solution as it enables a transducer designer to determine the transducer size that would induce maximum displacements at a given frequency. This is a key aspect in the transducer design as the excitation frequency is directly related to the damage type to be detected.

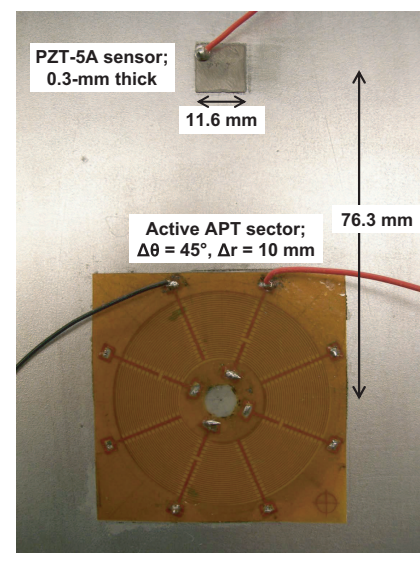

(a)

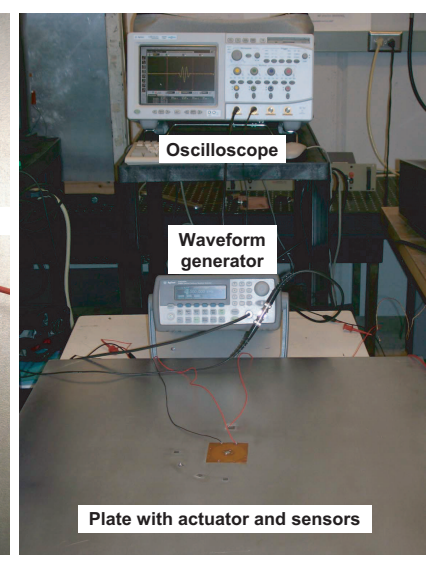

(b)

Figure 5. Experimental Setup: (a) CLoVER actuator and sensor detail; (b) Overall arrangement.

\section{A. Experimental Setup}

In order to verify the analytical model presented in section III, a $3.2 \mathrm{~mm}$-thick square aluminum 5005 plate was used in the experimental tests. The plate thickness was selected so that the effect of the actuator's dynamics on the substrate system could be neglected. Past studies have shown successful results employing a similar arrangement. ${ }^{12,13}$ The plate had a side length of $0.7 \mathrm{~m}$. In an effort to excite pure symmetric and antisymmetric modes over a wide frequency range, one CLoVER transducer was bonded on each surface of the plate at its geometric center. The sectors used had a radial dimension $\Delta \mathrm{r}=1.0 \mathrm{~cm}\left(R_{O}=2.5 \mathrm{~cm}\right.$, $R_{I}=1.5 \mathrm{~cm}$ ) and an azimuthal $\Delta \theta=45^{\circ}$. The symmetric mode was obtained by exciting both actuators in phase, while the antisymmetric mode resulted from exciting them out of phase. The induced wave field was recorded using a square piezoceramic sensor with a side length of $1.16 \mathrm{~mm}$ and a thickness of $0.3 \mathrm{~mm}$. The sensor was located along the transducer's centerline at a radial distance of $76.3 \mathrm{~mm}$. This position was selected based on the plate's dimension, so that boundary reflections were avoided, thereby satisfying the infinite plate assumption. The actuators and the sensor were bonded by applying a thin layer of Epotek 301 bonding agent and allowing it to cure for 24 hours. The actuator and sensor arrangement used is illustrated in Fig. 5(a). Each actuator was excited with a 3.5-cycle Hann-modulated toneburst signal using an arbitrary waveform generator (Agilent 33220A). A digital oscilloscope(Agilent Infinuum 54831DSO) was used to monitor and acquire the voltage signal from the sensor, which was averaged over 64 samples at a sampling rate of 10 mega samples per second. The overall set up is illustrated in Fig. 5(b).

\section{B. Results and Discussion}

The sensor frequency response was measured experimentally and simulated using the analytical model in section III. A sample of these results is shown in Fig. 6, which shows the time history predicted by the analytical model is in good agreement with the data recorded from the piezoelectric sensor. The material properties used for the aluminum alloy in the computations are summarized in Table 1. The figure illustrates the results for the $\mathrm{A}_{0}$ mode at $65 \mathrm{kHz}$. It can be noted that the amplitude and time of arrival of each peak are 
very well captured by the model, as the error in the time of arrival is less than $2 \%$ while the largest amplitude difference (which occurs for the final secondary peak) is less than $8 \%$ of the peak-to-peak amplitude of the pulse.

Table 1. Aluminum substrate material properties

\begin{tabular}{cc} 
Property & Value \\
\hline $\mathrm{E}$ & $68.9 \mathrm{GPa}$ \\
$\nu$ & 0.33 \\
$\rho$ & $2700 \mathrm{~kg} / \mathrm{m}^{3}$
\end{tabular}

Several time histories, similar to the one presented in Fig. 6, were recorded over different frequencies for both the symmetric and antisymmetric modes. These results are summarized in Fig. 7 where each data set has been normalized by its maximum value. The experimental data points represent average values, while the error bars represent three times the peak-to-peak standard deviation. This uncertainty was found by taking 64 averages at each frequency. The analytical predictions were obtained using Eq. (2). It can be seen that there is good agreement between the analytical prediction and the experimental data, especially for the antisymmetric mode where the differences between the theoretical and experimental results are within the experimental uncertainty for most of the points. It can also be appreciated that there is good correlation between both results for the symmetric mode until a frequency of approximately $275 \mathrm{kHz}$. Beyond this frequency, the EMI generated by the CLoVER sector is very strong and its interaction with the excitation pulse is very significant. Consequently, the peak-to-peak amplitude of the response recorded had to be manually measured according to the expected time of arrival of the pulse, calculated based on its group velocity at each frequency. The EMI results from the rapidly changing electric currents flowing through the interdigitated electrode fingers of the transducer. An important area for further development is the shielding of the transducer and sensor to this type of interference.

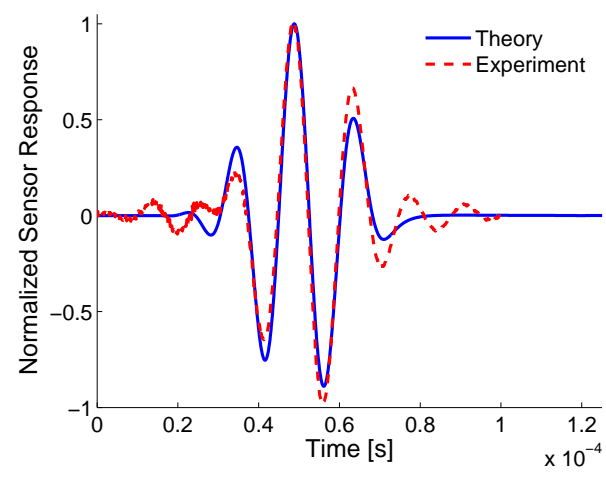

Figure 6. Time-history comparison for $\mathrm{A}_{0}$ mode at $65 \mathrm{kHz}$.

\section{Laser Vibrometer Experiments}

In this section, the non-contact technique of laser vibrometry is used to investigate the GW field induced by a CLoVER sector. This method is an important complement to the sensor-based experiments presented in the previous section as it is able to provide information on the spatial variations of the GW field. The section begins with a brief overview of the operating principles of the scanning laser vibrometer and a description of the experimental setup used. A comprehensive set of results are subsequently presented to verify the spatial and temporal accuracy of the theoretical solution. 


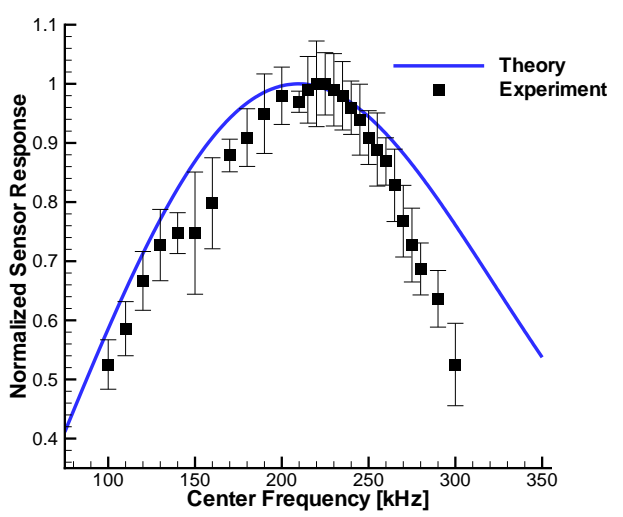

(a): $\mathbf{S}_{0}$ mode

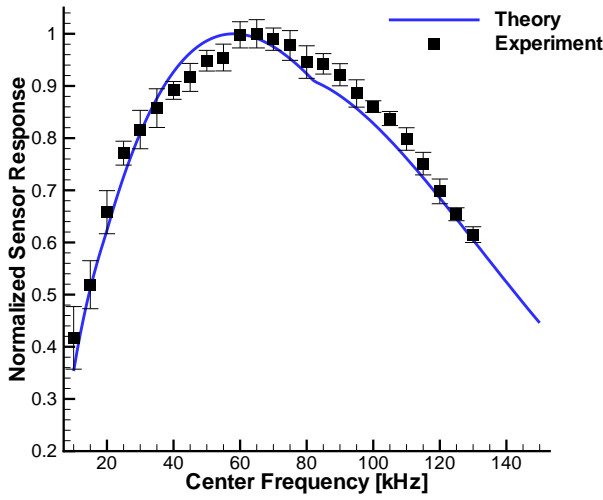

(b): $\mathbf{A}_{0}$ mode

Figure 7. Comparison between analytical and experimental frequency response results

\section{A. Setup and Laser Vibrometer Operation}

The key instrument used in these studies was a Polytec PSV-400 scanning laser vibrometer. This system is composed of the PSV-I-400 scanning head, the OFV-5000 controller, the PSV-E-401 junction box, and a data management system. The light source used in the PSV-400 is a helium neon laser that provides a linear polarized beam. ${ }^{25}$ The vibrometer system is able to measure the out-of-plane velocities by measuring the difference in path lengths between a reference beam and an object beam that is backscattered from the surface under inspection. The PSV-I-400 scanning head houses a high sensitivity vibrometer sensor, a high precision scan unit, and a color video camera used to manipulate the laser and scan points in the PSV software. The OFV-5000 controller is able to decode the interference signal of the object and reference beams, while the PSV-E-401 junction box provides an interface for the scanning head, controller, and data management system as well as several input channels for triggering and signal generator output. Finally, the data management system houses the PSV software used to operate the laser vibrometer.

The scanning head was supported using a tripod and maintained at a distance of $915 \mathrm{~mm}$ from the scanning surface for most of the experimental tests conducted. This distance was selected to operate at a laser visibility maximum. ${ }^{25}$ An important consideration when taking laser vibrometer measurements is that the scanning angles be small enough so that contributions from the in-plane components remain negligible. The horizontal and vertical scanning angles are defined in Fig. 8(a). Furthermore, large scanning angles $\left(\sim 10^{0}\right)$ are expected to reduce the signal-to-noise ratio. The work of Leong et. al. ${ }^{18}$ provided important insight into this issue. They showed that the vertical scan angle should be kept below four degrees in order to maintain a consistent signal to noise ratio, while the amplitudes of the displacements measured were mostly insensitive to variations in the horizontal scan angle. Consequently, the plate under inspection was arranged so that the vertical scan angle did not exceed the four-degree limit.

The test specimen consisted of the same isotropic plate and CLoVER transducer arrangement described in section A of the sensor-based experiments. The surface to be scanned was lightly sanded using 120 grade sand paper to enhance its backscattering behavior. As the laser vibrometer is primarily sensitive to the out-of-plane displacement component, the center frequency of the excitation pulse used was maintained at $65 \mathrm{kHz}$. It was shown earlier through sensor-response experiments that the $\mathrm{A}_{0}$ mode is maximized near this frequency. This mode was selected as it is well known that it has a predominantly out-of-plane component. In this case, the excitation pulse was amplified using a Trek PZD2000 high power amplifier which provided a fixed gain of 200. The input voltage used in this set of tests was higher than in the sensor tests to obtain a high signal-to-noise ratio in the laser vibrometer, and varied from 200 to $800 V_{p-p}$. The overall experimental setup is illustrated in Fig. 8(b). 


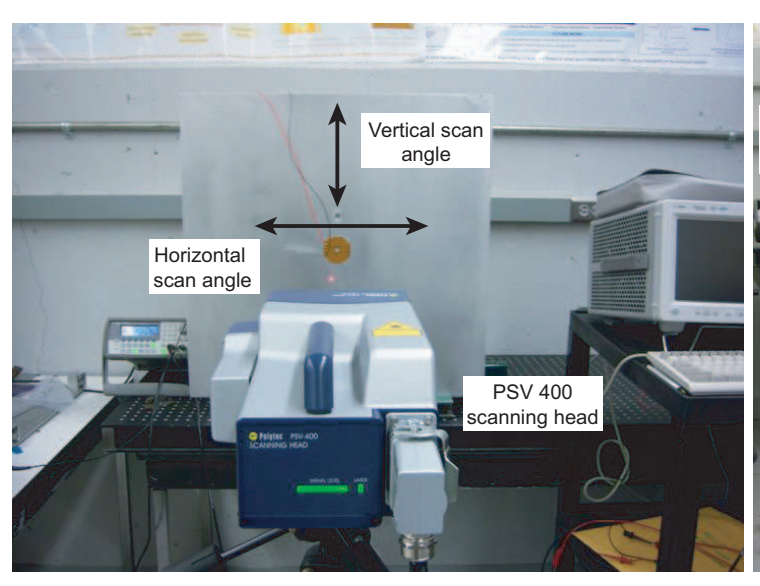

(a)

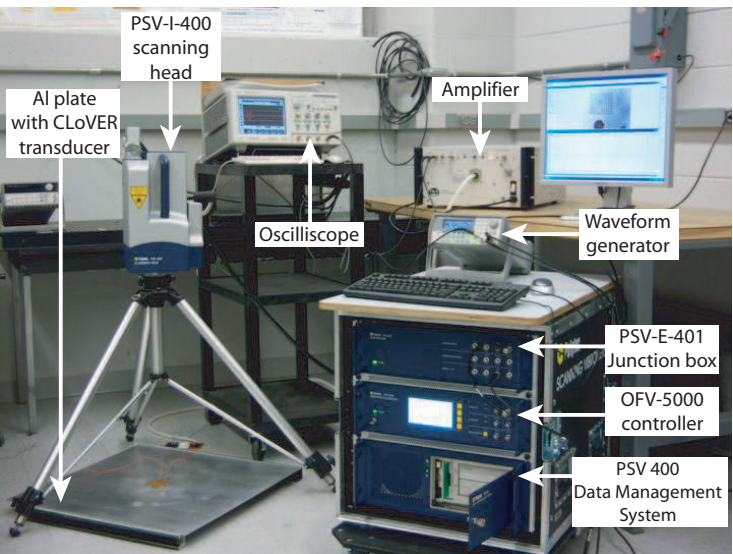

(b)

Figure 8. (a) Definition of horizontal and vertical scan angles; (b) Experimental setup used in laser vibrometer tests.

\section{B. Results and Discussion}

The first set of tests consisted of evaluating the time history performance of the solution. Three points were selected at a fixed distance of $3.4 R_{O}$ and various azimuthal locations, as shown in Fig. 9(a). The time of arrival is close for both solutions (within $5 \%$ ) as shown in Figs. 9(b)-(d). The shape of the toneburst signal is also well captured although there are some differences in the relative amplitude of both the main and secondary peaks. The largest of these differences occurs for the time history recorded at $72^{0}$ and corresponds to approximately $30 \%$ of the peak-to-peak amplitude of the excitation pulse. As previously mentioned, the excitation voltage used in these experiments was significantly larger than that used in the sensor-based experiments, partially to obtain a good signal to noise ratio. A larger voltage input generates larger strains in the transducer, and it is possible that this results in a more pronounced contribution from the transducer dynamics which have been neglected in the theoretical solution. It should be noted that there are additional reasons for selecting a higher excitation voltage in this set of experiments. First, in this case only one transducer is being used in contrast to the two used in the previous experiments. This was done because in preliminary testing it was found that due to the sensitivity of the laser beam, minor misalignments in the placement of the transducers $(\sim 2 \mathrm{~mm})$ significantly affected the shape of the propagating pulse. It is likely that the sensor is not as sensitive to these differences as it is primarily excited by the weaker (in the $\mathrm{A}_{0}$ case) in-plane displacements. In addition, the piezoceramic fibers used in the CLoVER transducer are relatively thin. Finally, the antisymmetric mode is being excited using shear tractions in the plane of the substrate. It has been previously reported ${ }^{4}$ that, due to this mode's strong out-of-plane component, transducers that apply surface tractions normal to the surface are preferred for $\mathrm{A}_{0}$ excitation when employing a single device.

The second set of tests consisted of evaluating the decay in the propagating pulse amplitude with radial distance. It is important to accurately capture this parameter in the theoretical solution as it is key in determining the inspection distance that can be achieved with the transducer. In this case, several radial positions oriented along the centerline of a CLoVER sector were selected and the laser was used to measure the time history. The peak-to-peak amplitude obtained from these measurements were then compared to similar results obtained using the theoretical model. This result is shown in Fig. 10, where the results have been normalized by the mean peak-to-peak amplitude in each data set. As in previous cases, the error bars represent three times the standard deviation. This uncertainty was found by repeating each measurement three times, collecting 64 averages in each test. The figure shows that the experimental and theoretical results are in excellent agreement, with the differences bewteen them staying within the experimental uncertainty for all the measurement points.

Another important consideration is the azimuthal distribution of the induced GW field. This parameter indicates the directionality achieved by a CLoVER sector. As in the radial decay study, the peak-to-peak displacement amplitude was measured at several azimuthal and radial locations as shown in Fig. 11(a). In 


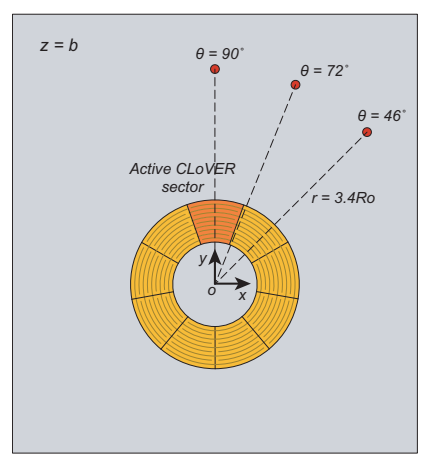

(a)

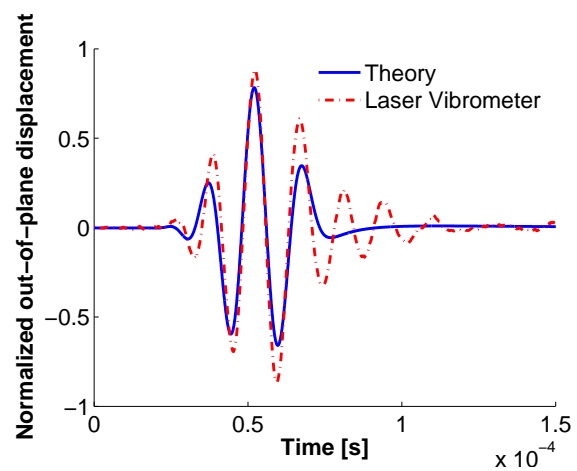

(c)

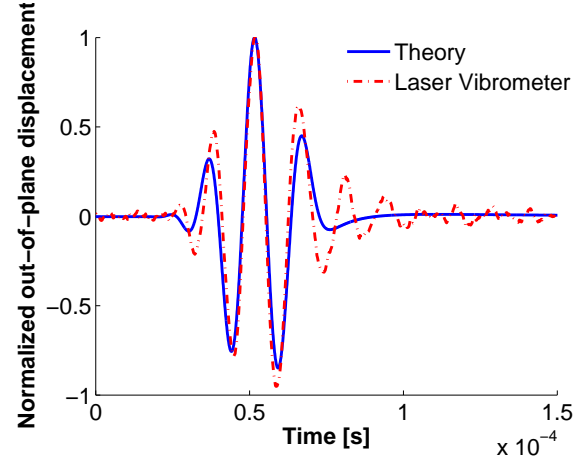

(b)

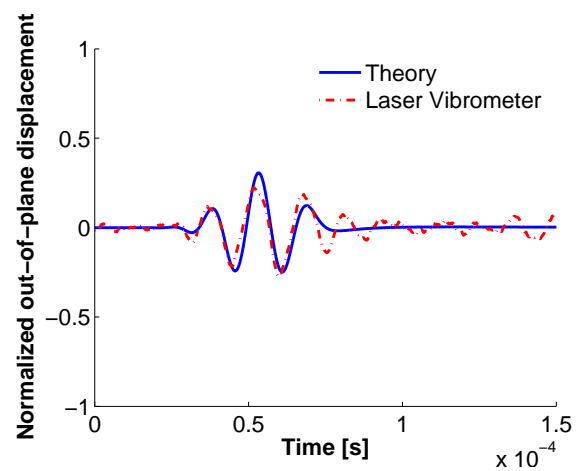

(d)

Figure 9. (a) Schematic illustrating distribution of measurement points; Time history comparison at $r=3.2$ $R_{0}$ and: (b) $\theta=\mathbf{9 0}^{0}$; (c) $\theta=\mathbf{7 2}^{0}$; (d) $\theta=\mathbf{4 6}^{\circ}$.

this case, a complete $360^{\circ}$ range was scanned using a grid consisting of 60 azimuthal points. In addition, three radial locations were chosen to investigate the evolution of the azimuthal distribution with radial distance. The measurements were repeated three different times with each set consisting of 64 averages. The displacement field was calculated theoretically for the same radial locations using a finer grid of azimuthal points. These results are compared in several polar plots shown in Figs. 11(b)-(d). In these figures, the transducer centerline coincides with the $90^{\circ}$ direction, and the peak-to-peak amplitude is represented by the radial distance from the origin for each azimuthal location. The uncertainty for each point is represented by two finer lines surrounding the experimental points which correspond to three times the standard deviation.

The results show that the azimuthal distribution is captured well by the model. The regions where the largest disagreements are observed correspond to normal directions from the transducer centerline where the GW field is smallest, which in turn decreases the signal-to-noise ratio in the laser vibrometer resulting in large standard deviations. Some differences are also observed for the wave field induced in the opposite direction, i.e., towards the $270^{\circ}$ direction, especially for larger radial distances. The largest error in this case is in the order of $23 \%$ and it occurs for the farthest radial position tested, at an azimuthal location of approximately $300^{\circ}$. It is likely that this disagreement is partly due to the presence of additional CLoVER sectors which are not accounted for in the theoretical solution. These additional transducers introduce concentrated masses which act as very small GW scatterers. As the radial position is increased, a larger portion of the wave field interacts with the additional sectors, which may explain the increasing difference with larger distance. It can also be appreciated from the figures that as the radial position is increased, the main displacement lobe becomes wider, which is consistent with the expectation that in the far field the wavefront would tend to a uniform circular front. ${ }^{26}$

The final set of tests consisted of a full-field scan to visually compare the temporal and spatial evolution of the GW field induced by a CLoVER sector. The scanning grid used in this case consisted of 25 radial points distributed over a seven centimeter range, which provided approximately three wavelengths with seven 


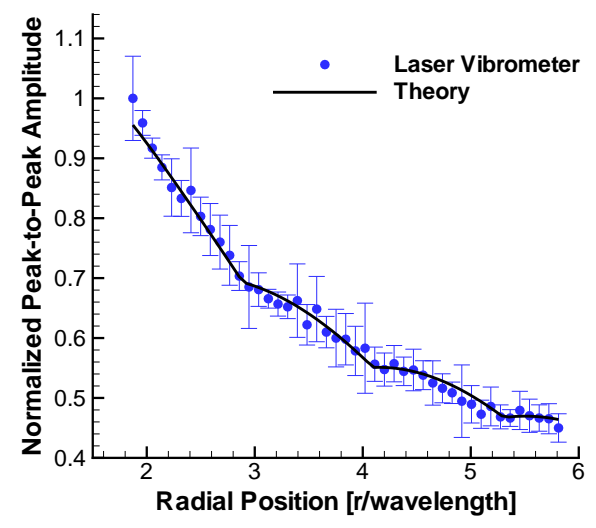

Figure 10. Comparison between theoretical and laser vibrometer results for peak-to-peak amplitude.

points per wavelength. A complete $360^{\circ}$ range was covered using an azimuthal grid consisting of 40 points. The starting radial position for the measurements was selected at one centimeter from the radial edge of the CLoVER sector to avoid scanning over pieces of kapton film that remain attached to house the electrode bus of the transducer. Similarly, the radial positions scanned excluded points near the origin as a piezoelectric sensor was bonded in this area. These results are summarized in Figs. 12 through 14, which show very good correlation between the theoretical model and the experiment. The directionality of the transducer is clearly visible, as the induced displacements remain primarily within the azimuthal span of the CLoVER sector.

\section{Damage Interrogation using CLoVER Transducers}

This section provides a preliminary demonstration of the damage interrogation approach proposed with CLoVER transducers. The experimental setup used is first discussed along with background information on damage scattering from structural defects, followed by the experimental results.

\section{A. Setup and Background}

Four sectors (labeled CLoVER 1 through CLoVER 4) were used to simulate the damage interrogation approach proposed with the CLoVER transducer, as shown in Fig. 15. Each of these was excited using a 3.5 Hann-modulated toneburst with a center frequency of $65 \mathrm{kHz}$. The peak-to-peak voltage input was slightly different for each sector to ensure that the peak-to-peak amplitude of the main pulse received by the sensor was similar for all of them. The only parameter not simulated in this study is the time delay that exists between alternating sectors, as they were activated manually. However, this is expected to be a fixed parameter chosen so that boundary reflections are completely attenuated.

A through-thickness hole with a diameter of $4.8 \mathrm{~mm}$ was drilled at a radial distance of $4 R_{O}$ away from the radial edge of one CLoVER sector to simulate structural damage, as also shown in Fig. 15. The same isotropic plate structure and CLoVER transducer used in the previous cases was employed here. The diameter of the hole to wavelength ratio was approximately 0.25 . This dimension was selected so that the reflections from the front and back of the hole would interfere constructively as shown by Diligent for the case of part-depth holes. ${ }^{27,28}$ However, it is expected that the reflection amplitude from a through-hole should increase monotonically with dimension as also discussed by Diligent et. al. ${ }^{29}$ The sensor response was measured using a circular piezoelectric sensor $(0.2 \mathrm{~mm}$ thick) bonded at the center of the CLoVER transducer. This study uses the pulse-echo mode, where a signal corresponding to the pristine condition of the structure is subtracted from one representing the damaged condition to expose reflections from any existing damage sites. As our goal is concerned with quantifying the spatial variations of the reflections from the damage site, its location is assumed to be known a priori. This allows the group velocity of the $\mathrm{A}_{0}$ mode to be used along with the damage location to estimate the time at which reflections are expected according to: 


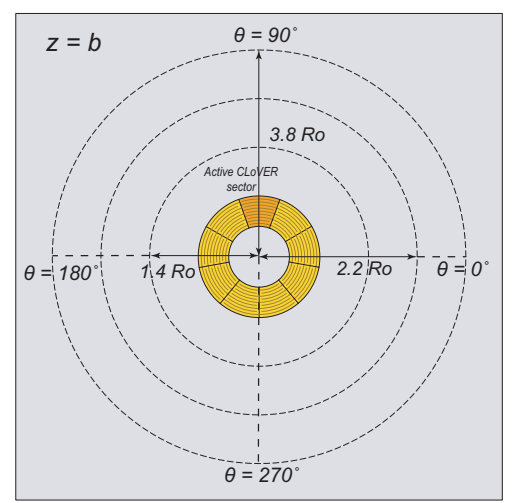

(a)

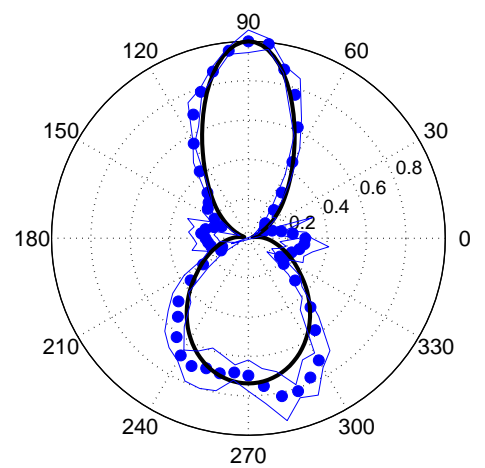

(c)

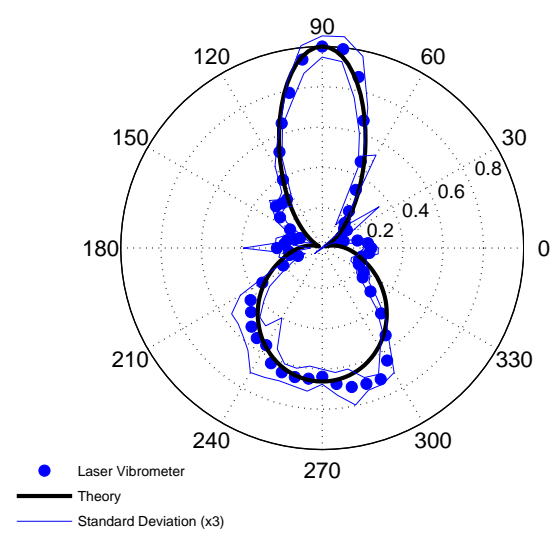

(b)

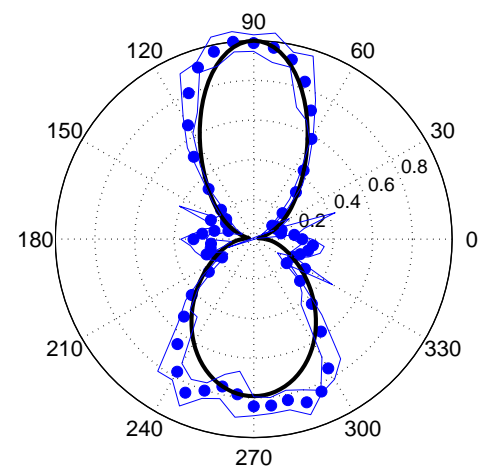

(d)

Figure 11. (a) Schematic showing location of the scan points used in azimuthal comparison tests; Comparison of peak-to-peak amplitudes at: (b) $\mathbf{r}=1.4 R_{O} ;(\mathbf{c}) \mathbf{r}=2.2 R_{O} ;(\mathbf{d}) \mathbf{r}=3.8 R_{O}$.

$$
t_{r}=\frac{2 d_{h}+R_{O}}{c_{g}}+\frac{T_{p}}{2}
$$

where the center of the reflected signal is tracked by adding one half the excitation pulse's period. In preliminary studies it was found that the times of arrival obtained assuming the reflections originated at the front of the hole were very similar to those obtained assuming they originated at the back. Consequently, only the value corresponding to the front of the hole was used to track the damage reflections. It has been previously reported that the $\mathrm{S}_{0}$ mode is more sensitive to through-the-thickness damage due to its dominant in-plane components. ${ }^{4,21}$ However, in order to excite a pure symmetric mode two transducers are needed on each surface of the substrate as explained in an earlier section. Some of the CLoVER sectors on one of the transducers employed were unavailable for testing as they had sustained damage during earlier electric poling tests, therefore making the excitation of symmetric modes for all sectors impossible. Consequently, the present study uses the $A_{0}$ mode to provide a concept demonstration of the proposed damage interrogation approach.

\section{B. Results and Discussion}

Figure 16(a) shows a comparison between signals corresponding to the pristine and damaged conditions of the structure. The regions where damage reflections are expected is delimited by the incoming excitation 


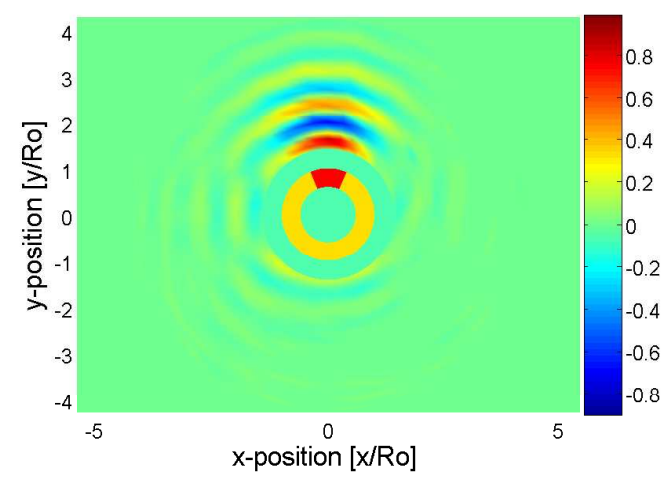

(a): Experiment

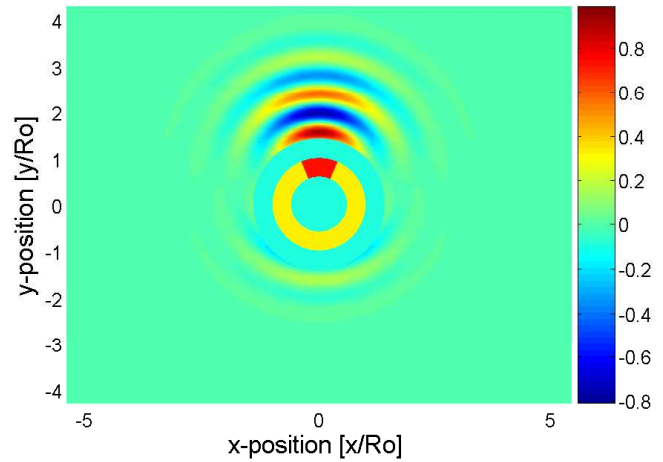

(b): Theory

Figure 12. Full-field comparison between laser vibrometer and theoretical solution at time $\mathrm{t}=35 \mu$ s.

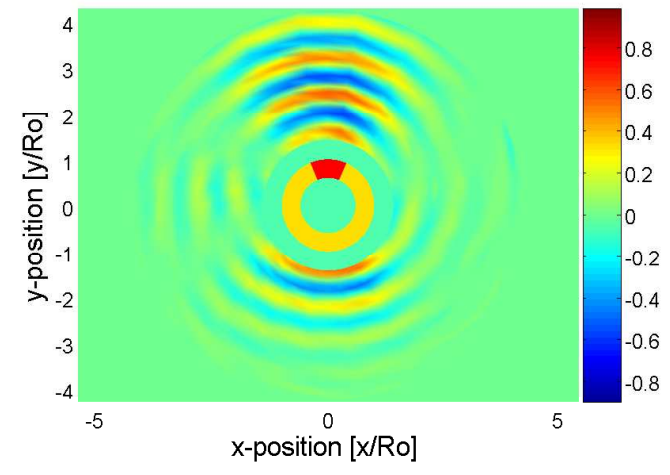

(a): Experiment

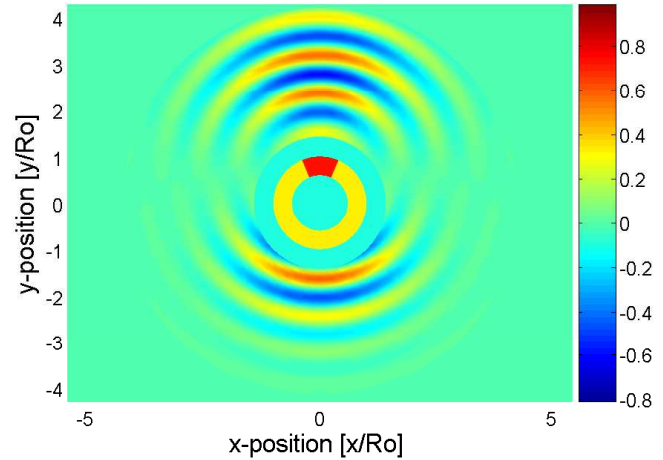

(b): Theory

Figure 13. Full-field comparison between laser vibrometer and theoretical solution at time $\mathbf{t}=50 \mu \mathrm{s}$.

pulse and the reflections from the plate boundaries. Figure 16(b) shows a sample difference signal where the expected time of arrival for defect reflections is highlighted. Similar signals were recorded for all CLoVER sectors used, and the peak-to-peak value in the region where the reflection is expected was measured in each case. This result is summarized in Fig. 17, which shows the peak-to-peak amplitude recorded for each CLoVER sector. The results presented correspond to average values, while the error bars represent three times the standard deviation. Two measurements were taken with each sector and 128 averages were collected in each of these.

The maximum amplitude is recorded when CLoVER 2 is activated, which is the expected result as the damage is located along this sector's centerline. Furthermore, the reflection amplitude for the remaining sectors decreases with increasing index (for sectors 3 and 4), as the centerline of these transducers moves farther away from the damage location. This result illustrates the usefulness of the interrogation approach attainable with the CLoVER transducer, as with prior knowledge of the device's configuration (namely the azimuthal direction of each sector), valuable information about the presence of damage can be inferred.

\section{Summary and Conclusions}

This paper presented several experimental studies on the guided wave (GW) field induced by the Composite Long-range Variable-direction Emitting Radar (CLoVER) transducer. This novel device has been 


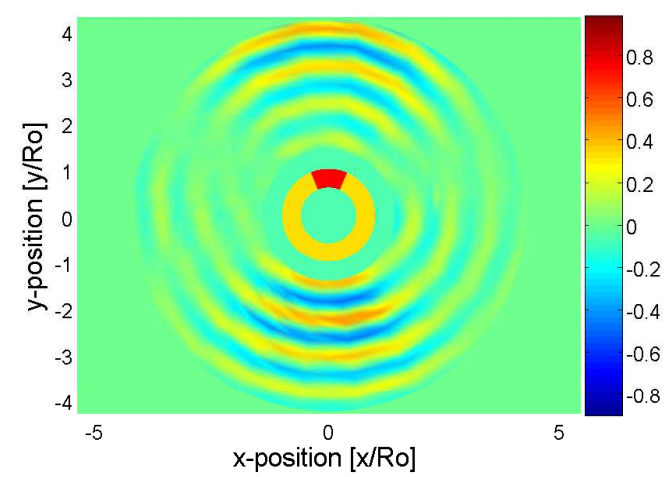

(a): Experiment

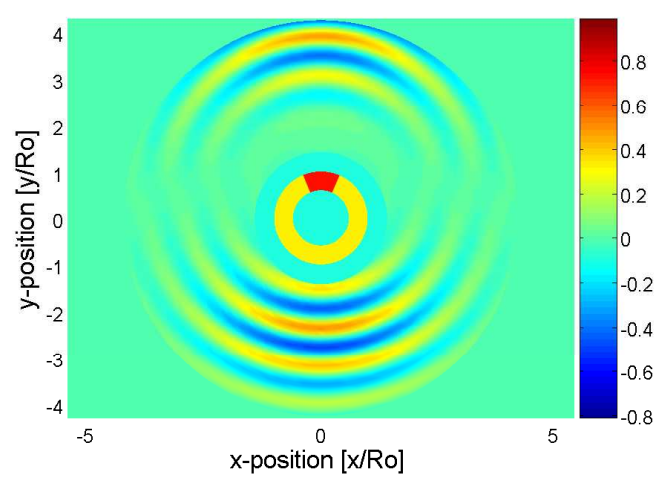

(b): Theory

Figure 14. Full-field comparison between laser vibrometer and theoretical solution at time $\mathbf{t}=65 \mu \mathrm{s}$.

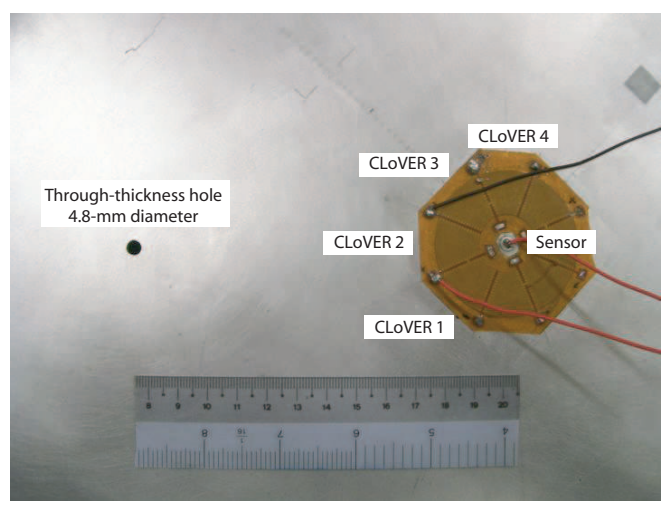

Figure 15. Detail of the structural damage introduced for damage interrogation approach demonstration.

introduced by the authors as an alternative concept for damage interrogation and GW excitation in structural health monitoring (SHM) systems. This transducer has an overall ring geometry, but is composed of wedge-shaped anisotropic piezocomposite sectors that can be individually excited to interrogate the structure along a particular direction from a central location. The multiple advantages offered by this new design were outlined, in particular as they improve several shortcomings of current transducer configurations. Each CLoVER sector is able to induce GW fields with larger amplitudes than a similarly sized ring geometry for the same electric current inputs, thereby achieving efficient excitation. Moreover, the design of its electrode pattern gives each CLoVER sector the ability to act as independent actuator and sensor element which reduces the number of transducers needed for inspection. Furthermore, these radial subdivisions may be used to excite specific GW modes. A theoretical model for GW excitation by a CLoVER sector previously presented by the authors was reviewed, and a combination of sensor-based and laser vibrometer-based experiments were conducted to verify its accuracy. A comprehensive set of results was presented to demonstrate the accuracy of the solution in the time, frequency, and spatial domains. Several laser vibrometer studies further verified the directionality achieved by this transducer geometry. The damage interrogation approach proposed with the CLoVER transducer was experimentally demonstrated using simulated structural damage, and it was shown that the damage reflections are maximized when the sector whose centerline is aligned with the damage is activated. This shows the potential of this transducer for efficient damage interrogation in GW SHM systems. 



(a)

(b)

Figure 16. (a) Comparison between pristine and damaged sensor response signals; (b) Difference signal between pristine and damaged conditions.

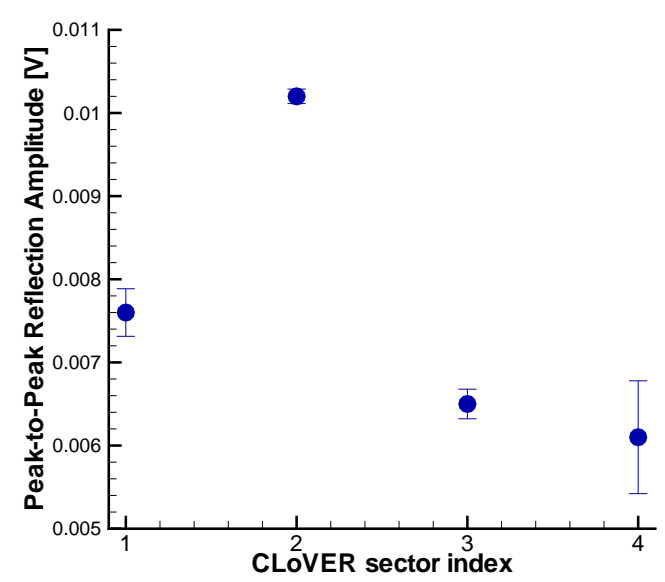

Figure 17. Comparison of reflection amplitudes among different CLoVER sectors.

\section{Acknowledgements}

This work was supported by the Air Force Office of Scientific Research under grants FA9550-06-1-0071 and FA9550-07-1-0522 with Dr. Victor Giurgiutiu as the technical monitor. Suggestions from Dr. Ajay Raghavan (University of Michigan, presently at Metis Design Corporation) regarding the experimental setup for the sensor response experiments are thankfully acknowledged. 


\section{References}

${ }^{1}$ Raghavan, A. and Cesnik, C. E. S., "Review of guided-wave based Structural Health Monitoring," The Shock and Vibration Digest, Vol. 39, No. 2, 2007, pp. 91-114.

${ }^{2}$ Bent, A. A. and Hagood, N. W., "Anisotropic Actuation with Piezoelectric Fiber Composites," Journal of Intelligent Materials, Systems and Structures, Vol. 6, No. 3, 1995, pp. 338-349.

${ }^{3}$ Wilkie, W. K., High, J. W., and Bockman, J., "Reliability Testing of NASA piezocomposite actuators," Proceedings of the 8th International Conference on New Actuators, Bremen, Germany, 2002.

${ }^{4}$ Wilcox, P., Lowe, M., and Cawley, P., "Lamb and SH wave transducer arrays for the inspection of large areas of thick plates," AIP Conference Proceedings Review of Progress in Quantitative Nondestructive Evaluation, , No. 509A, 2000, pp. 1049-1056.

${ }^{5}$ Fromme, P., Wilcox, P. D., Lowe, M. J. S., and Cawley, P., "On the development and testing of a guided ultrasonic wave array for structural health monitoring," IEEE Transactions on Ultrasonics, Ferroelectrics, and Frequency Control, Vol. 53, No. 4, 2006, pp. 777-785.

${ }^{6} \mathrm{Yu}, \mathrm{L}$. and Giurgiutiu, V., "Design, implementation and comparison of guided wave phased arrays using embedded piezoelectric wafer active sensors for structural health monitoring," Proceedings of the SPIE, Vol. 6173, 2006.

${ }^{7} \mathrm{Yu}$, L., Giurgiutiu, V., and Kendall, J. R., "Omnidirectional Guided Wave PWAS Phased Array for Thin-wall Structure Damage Detection," Proceedings of the SPIE, Vol. 6529, 2007.

${ }^{8}$ Zemmour, A. I., "The Hilbert-Huang Transform for Damage Detection in Plate Structures," M.S. Thesis, University of Maryland, 2006.

${ }^{9}$ Cesnik, C. E. S., "Integrated Vehicle Health Management System," Proceedings of the NASA Reusable Launch Vehicle URETI, Cleveland, Ohio, NASA Task 5c Annual Report, 2003.

${ }^{10}$ Salas, K. I. and Cesnik, C. E. S., "Design and Characterization of the CLoVER Transducer for Structural Health Monitoring," Proceedings of the SPIE, 2008.

${ }^{11}$ Salas, K. I., Cesnik, C. E. S., and Raghavan, A., "Modeling of Wedge-shaped Anisotropic Piezocomposite Transducer for Guided wave-based Structural Health Monitoring," Proceedings of the $15^{\text {th }}$ AIAA/ASME/AHS Adaptive Structures Conference, Honolulu, HI, 2007.

${ }^{12}$ Raghavan, A. and Cesnik, C. E. S., "Modeling of piezoelectric-based Lamb wave generation and sensing for structural health monitoring," Proceedings of the SPIE Symposium on Smart Structures and Materials / NDE 2004, 2004.

${ }^{13}$ Raghavan, A. and Cesnik, C. E. S., "Finite-dimensional piezoelectric transducer modeling for guided wave based structural health monitoring," Smart Materials and Structures, Vol. 14, No. 6, 2005, pp. 1448-1461.

${ }^{14}$ Raghavan, A. and Cesnik, C. E. S., "Guided-wave signal processing using chirplet matching pursuits and mode correlation for structural health monitoring," Smart Materials and Structures, Vol. 16, 2007, pp. 355-366.

${ }^{15}$ Raghavan, A. and Cesnik, C. E. S., "Studies on effects of elevated temperature for guided-wave structural health monitoring," Proceedings of the SPIE Symposium on Smart Structures and Materials / NDE 2007, Vol. 6529, 2007.

${ }^{16}$ Staszewski, W., Lee, B. C., Mallet, F., and Scarpa, F., "Structural health monitoring using scanning laser vibrometry: I. Lamb wave sensing," Smart Materials and Structures, Vol. 13, 2004, pp. 251-260.

${ }^{17}$ Mallet, A., Lee, B. C., Staszewski, W., and Scarpa, F., "Structural health monitoring using scanning laser vibrometry: II. Lamb waves for damage detection," Smart Materials and Structures, Vol. 13, 2004, pp. 261-269.

${ }^{18}$ Leong, W. H., Staszewski, W., Lee, B. C., and Scarpa, F., "Structural health monitoring using scanning laser vibrometry: III. Lamb waves for fatigue crack detection," Smart Materials and Structures, Vol. 14, 2005, pp. 1387-1395.

${ }^{19}$ Staszewski, W., Lee, B. C., and Traynor, R., "Fatigue crack detection in metallic structures with Lamb waves and 3D laser vibrometry," Smart Materials and Structures, Vol. 18, 2007, pp. 727-739.

${ }^{20}$ Wilcox, P., Lowe, M., and Cawley, P., "Mode and Transducer Selection for Long Range Lamb Wave Inspection," Journal of Intelligent Materials Systems and Structures, Vol. 12, 2001, pp. 553-565.

${ }^{21}$ Alleyne, D. and Cawley, P., "The interation of Lamb waves with defects," IEEE Transactions on Ultrasonics, Ferroelectrics, and Frequency Control, Vol. 39, No. 3, 1992, pp. 381-397.

${ }^{22}$ Wilkie, W. K. and High, J. W., "Method of Fabricating NASA-Standard Macro-Fiber Composite Piezoelectric Actuators," NASA/TM-2003-212427, NASA, 2003.

${ }^{23}$ Williams, R. B., Grimsley, B. W., Inman, D. J., and Wilkie, W. K., "Manufacturing and Cure Kinetics Modeling for Macro Fiber Composite Actuators," Journal of Reinforced Plastics and Composites, Vol. 23, No. 16, 2004, pp. 1741-1754.

${ }^{24}$ Wilkie, W. K., Inman, D. J., Lloyd, J. M., and High, J. W., "Anisotropic Laminar Piezocomposite Actuator incorporating machined PMN-PT Single-crystal fibers," Journal of Intelligent Materials Systems and Structures, Vol. 17, No. 1, 2006, pp. 1528.

${ }^{25}$ Polytec Scanning Laser Vibrometer PSV 400 Hardware Manual, Polytec GmbH, 2007.

${ }^{26}$ Graff, K., Wave Motion in Elastic Solids, Dover Publications, 1991.

${ }^{27}$ Diligent, O. and Lowe, M. J. S., "Reflection of the $\mathrm{S}_{0}$ Lamb mode from a flat bottom circular hole," Journal of Acoustical Society of America, Vol. 118, No. 5, 2005, pp. 2869-2879.

${ }^{28}$ Grahn, T., "Lamb wave scattering from a circular partly through-thickness hole in a plate," Wave Motion, Vol. 37, No. 1, 2003, pp. 63-80.

${ }^{29}$ Diligent, O., Grahn, T., Bostrom, A., Cawley, P., and Lowe, M. J. S., "The low-frequency reflection and scattering of the $\mathrm{S}_{0}$ Lamb mode from a circular through-thickness hole in a plate: Finite Element analytical and experimental studies," Journal of the Acoustical Society of America, Vol. 112, No. 6, 2002, pp. 2589-2601. 